\title{
Modulatory Effect of Eui-E-In-Tang on Serum Leptin Concentration in Obese Korean Female Adults: A Randomized Controlled Trial
}

\author{
Yun-Kyung Song, ${ }^{1}$ Ji-Young Lee, ${ }^{2}$ and Wansu Park ${ }^{2}$ \\ ${ }^{1}$ Department of Korean Rehabilitation Medicine, College of Korean Medicine, Gachon University, Seongnam 13120, Republic of Korea \\ ${ }^{2}$ Department of Pathology, College of Korean Medicine, Gachon University, Seongnam 13120, Republic of Korea
}

Correspondence should be addressed to Wansu Park; pws98@gachon.ac.kr

Received 27 February 2016; Accepted 30 June 2016

Academic Editor: Mariangela Rondanelli

Copyright (C) 2016 Yun-Kyung Song et al. This is an open access article distributed under the Creative Commons Attribution License, which permits unrestricted use, distribution, and reproduction in any medium, provided the original work is properly cited.

Background. Obesity is associated with chronic inflammation and cytokines. However, to date, the relationship between the serum levels of cytokines in obese individuals and taking herbal drugs remains largely unexplored. Methods. Serum cytokines were assessed by multiplex cytokine profiling assay. Serum samples of obese female Korean adults (obese group; $n=20$ ) as well as normal female Korean adults (normal group; $n=21$ ) were collected at the start and end of study period. Twenty obese female Korean adults were randomized to receive Eui-E-In-Tang (Eui-E-In-Tang group; $n=9$ ) at a daily dose of $9 \mathrm{~g}$ or a matched placebo (placebo group; $n=11$ ) for 12 weeks. Results. According to investigating serum cytokine levels at the start point of this study, the serum C-peptide, insulin, leptin, lipocalin-2, and adipsin levels in the obese group were found to be significantly higher than in the normal group. And the investigation of serum cytokine levels at the end point of this study demonstrated that mean serum leptin of Eui-E-In-Tang group was found to be significantly reduced $(P=0.037)$. Conclusions. This study provides preliminary evidence that Eui-E-In-Tang may exert immunomodulatory effect via altering the circulating concentration of leptin in Korean female adults.

\section{Introduction}

Obesity is a serious health problem worldwide. According to the World Health Organization (WHO) statistics, there were more than 1.9 billion overweight adults globally, of whom about 600 million were obese in 2014 [1].

Obesity is associated with an increased risk of lifethreatening diseases including diabetes, cardiovascular diseases, metabolic syndrome, osteoarthritis, gout, and certain types of cancer [2-4]. Adya et al. have already reported that obesity is a major health burden with an increased risk of cardiovascular morbidity and mortality [5]. dos Santos et al. have reported that inflammation, the process aimed at restoring homeostasis after an insult, can be more damaging than the insult itself if uncontrolled, excessive, or prolonged [6]. Specifically, in mediators of chronic inflammation, cytokine (cell signaling protein) has been becoming the impressive target to deal with obesity. Alvehus et al. have reported that obesity can be considered as a low-grade inflammatory condition, strongly linked to adverse metabolic outcomes [7]. It has been suggested that adipocytokine (adipokine) such as leptin is secreted by adipose tissue and concerned with the pathogenesis of obesity-associated complications $[8,9]$.

Yen et al. have reported that inflammation has been found to be an important characteristic of adipose tissue in obese subjects and obesity is also associated with compromised immune responses to infections, which have not been fully understood [10]. Schulte et al. have reported that cytokines are important pleiotropic regulators of the immune response, which have a crucial role in the complex pathophysiology underlying sepsis [11]. Recently, anti-inflammatory effects of herbal drugs used in Korea, China, and Japan have been reported continuously. Eui-E-In-Tang is one of herbal drugs which are used for obese individuals in Korea.

However, to date, the relationship between the serum levels of cytokines in obese individuals and taking herbal drug such as Eui-E-In-Tang remains largely unexplored, especially in Korean women. In this study, we investigated 
modulatory effects of Eui-E-In-Tang on serum concentrations of cytokines in obese female Korean adults.

\section{Materials and Methods}

2.1. Study Design. This was a randomized, double blind, placebo-controlled trial in which each patient received EuiE-In-Tang ( $9 \mathrm{~g} /$ day) or a matched placebo for 12 weeks. All studies were approved by Bioethic Institutional Review Board at Gachon University. All subjects gave written informed consent prior to participation.

2.2. Subjects. Thirty-six apparently healthy adult Korean females were recruited from the local communities of Incheon and neighboring areas as normal subjects (the normal group). Obesity was defined by body mass index (BMI) $\geq$ $30.0 \mathrm{~kg} / \mathrm{m}^{2}$. Thirty-six obese adult Korean females were selected and recruited from the local communities of Incheon and neighboring areas as obese subjects (the obese group). Thirty-one individuals were excluded if they were taking any prescription or over-the-counter medication including oral contraceptives, hormone replacement therapy, or psychiatric drugs or had any acute or chronic medical illnesses during the study. Finally, 21 normal subjects and 20 obese subjects were selected for serum cytokine profiling assay in the study.

2.3. Drugs. Eui-E-In-Tang and placebo drug capsules were provided by the Hanpoong Pharmaceutical Co., Ltd. (Seoul, Korea). The components of Eui-E-In-Tang capsule are shown in Table 1. The placebo capsules were made of cornstarch and manufactured identical to Eui-E-In-Tang capsules in terms of color, size, and shape by the same manufacturer.

2.4. Multiplex Cytokine Assay. Multiplex cytokine profiling assay (MILLIPLEX MAP Human Metabolic Panel, Millipore, Billerica, MA, USA) was used to simultaneously measure serum levels of 21 cytokines such as C-peptide, ghrelin, glucose-dependent insulinotropic polypeptide (GIP), glucagon-like peptide-1 (GLP-1), glucagon, interleukin- (IL-) $1 \beta$, IL-6, IL-8, insulin, leptin, monocyte chemotactic protein(MCP-) 1, pancreatic polypeptide (PP), peptide YY (PYY), tumor necrosis factor- (TNF-) $\alpha$, nerve growth factor (NGF), hepatocyte growth factor (HGF), adiponectin, lipocalin-2, resistin, adipsin, and total plasminogen activator inhibitor type-1 (PAI-1). Samples were read on a Bio-Plex 200 suspension array system (Bio-Rad, Hercules, CA, USA) using BioPlex Manager 5.0 software system (Bio-Rad). The standard curve range for each cytokine was from 3.2 to $10,000 \mathrm{pg} / \mathrm{mL}$.

2.5. Statistical Analysis. The results represent the mean \pm SD. Descriptive analyses for the normal and obese groups are done using the independent sample $t$-test. The serum cytokine levels of the normal and obese groups were also compared via an independent sample $t$ test. Of them, C-peptide, insulin, leptin, HGF, adiponectin, lipocalin-2, adipsin, and total PAI1-all of which showed significant differences between the two groups-were compared with a univariate analysis of covariance. Correlation coefficients were calculated using Spearman's test. A paired $t$-test was used to compare serum
TABle 1: Composition of Eui-E-In-Tang.

\begin{tabular}{lc}
\hline Herbal medicines & Ratio \\
\hline Coicis Semen & 10 \\
Angelicae Gigantis Radix & 4 \\
Atractylodis Rhizoma Alba & 4 \\
Ephedrae Herba & 4 \\
Cinnamomi Ramulus & 3 \\
Paeoniae Radix & 3 \\
Glycyrrhizae Radix & 2 \\
\hline
\end{tabular}

cytokine levels before and after Eui-E-In-Tang therapy. All tests were two-tailed at a probability level of 0.05 . All analyses were performed using SPSS software 19.0 (SPSS Inc., Chicago, IL, USA).

\section{Results}

Table 2 presents the demographic factors, height, weight, and body mass index of the individuals in the normal and obese groups. We also compared vascular risk factors between the normal and obese group. Significant differences in the serum glutamate-pyruvate transaminase (SGPT), alkaline phosphatase, gamma-glutamyl transpeptidase (GGTP), total cholesterol, high-density lipoprotein (HDL) cholesterol, triglyceride, uric acid, glucose, C-reactive protein (CRP), and white blood cell (WBC) levels were observed between the two groups.

Among the 21 cytokines, the number of samples with detectable levels and the range $(\mathrm{pg} / \mathrm{mL})$ varied according to the tested cytokines. Data of cytokines which were detected to be $>80 \%$ of the total samples were used for further analysis and presented in Table 3. Data of cytokines included Cpeptide, GIP, insulin, leptin, MCP-1, PP, IL-8, HGF, adiponectin, lipocalin-2, resistin, adipsin, and total PAI-1. Among the 13 cytokines analyzed, the serum C-peptide, insulin, leptin, HGF, lipocalin, adipsin, and total PAI-1 levels of the obese group were found to be significantly higher than those of the normal group $(t=-5.104, P<0.001 ; t=-3.906$, $P=0.001 ; t=-6.777, P<0.001 ; t=-2.753, P=0.01$; $t=-2.062, P=0.046 ; t=-3.723, P=0.001$; and $t=-3.441$, $P=0.001$, resp.), whereas the serum adiponectin level of the obese group was found to be lower than that of the normal group ( $t=2.77, P=0.009$ ) (Table 3 ). After adjusting for age, height, SGPT, alkaline phosphatase, GGTP, total cholesterol, HDL cholesterol, triglyceride, uric acid, glucose, CRP, and WBC, the serum C-peptide, insulin, leptin, lipocalin-2, and adipsin levels in the obese group were found to be significantly higher than in the normal group $(F=4.673, P=0.04$; $F=4.358, P=0.046 ; F=28.469, P<0.001 ; F=7.236$, $P=0.012 ; F=5.37, P=0.028$, resp.).

Table 4 presents correlations among serum cytokines in the overall population. C-peptide was significantly correlated with insulin, leptin, IL-8, HGF, adiponectin, adipsin, and total PAI-1. GIP was significantly correlated with insulin, adiponectin, and lipocalin-2. Insulin was significantly correlated with leptin, HGF, adipsin, and total PAI-1. Leptin was 
TABLE 2: Demographic characteristics and obesity-related scale scores of participants.

\begin{tabular}{|c|c|c|c|c|}
\hline & Normal group ( $n=21$, female) & Obese group ( $n=20$, female $)$ & $t$ & $P$ \\
\hline Age (year) & $36.7 \pm 11.1$ & $41.4 \pm 9.5$ & -1.436 & 0.159 \\
\hline Height $(\mathrm{cm})$ & $161.5 \pm 4.7$ & $160.2 \pm 6.2$ & 0.796 & 0.431 \\
\hline Weight $(\mathrm{kg})$ & $53.8 \pm 4.4$ & $87.6 \pm 12.2$ & -11.729 & $<0.001$ \\
\hline Body mass index (BMI) & $20.7 \pm 1.5$ & $34.0 \pm 3.1$ & -17.397 & $<0.001$ \\
\hline Total protein $(\mathrm{g} / \mathrm{dL})$ & $7.5 \pm 0.3$ & $7.3 \pm 0.5$ & 1.018 & 0.315 \\
\hline Albumin $(\mathrm{g} / \mathrm{dL})$ & $4.7 \pm 0.2$ & $4.6 \pm 0.2$ & 1.419 & 0.164 \\
\hline Total bilirubin (g/dL) & $0.7 \pm 0.3$ & $0.6 \pm 0.3$ & 1.257 & 0.216 \\
\hline SGOT (U/L) & $19.2 \pm 6.8$ & $24.4 \pm 10.1$ & -1.939 & 0.06 \\
\hline SGPT (U/L) & $15.4 \pm 8.9$ & $25.0 \pm 11.5$ & -2.971 & 0.005 \\
\hline Alkaline phosphatase (U/L) & $49.6 \pm 14.3$ & $67.4 \pm 16.4$ & -3.691 & 0.001 \\
\hline GGTP (U/L) & $14.4 \pm 6.8$ & $33.0 \pm 28.6$ & -2.839 & 0.01 \\
\hline Creatine phosphokinase (U/L) & $111.4 \pm 123.8$ & $98.8 \pm 66.6$ & 0.404 & 0.688 \\
\hline Total cholesterol (mg/dL) & $174.1 \pm 24.3$ & $201.7 \pm 46.7$ & -2.359 & 0.025 \\
\hline HDL cholesterol (mg/dL) & $62.1 \pm 14.5$ & $53.2 \pm 13.0$ & 2.061 & 0.046 \\
\hline Triglyceride (mg/dL) & $74.8 \pm 32.8$ & $133.5 \pm 60.7$ & -3.822 & 0.001 \\
\hline Uric acid (mg/dL) & $4.4 \pm 0.8$ & $5.6 \pm 1.3$ & -3.675 & 0.001 \\
\hline Blood urea nitrogen $(\mathrm{mg} / \mathrm{dL})$ & $11.8 \pm 2.4$ & $12.8 \pm 3.7$ & -1.045 & 0.303 \\
\hline Creatinine $(\mathrm{mg} / \mathrm{dL})$ & $0.7 \pm 0.1$ & $0.7 \pm 0.1$ & -0.737 & 0.465 \\
\hline Glucose (mg/dL) & $85.8 \pm 9.1$ & $93.4 \pm 10.2$ & -2.512 & 0.016 \\
\hline $\mathrm{CRP}(\mathrm{mg} / \mathrm{dL})$ & $0.0 \pm 0.1$ & $0.3 \pm 0.5$ & -2.533 & 0.02 \\
\hline Free T4 (ng/dL) & $1.2 \pm 0.1$ & $1.2 \pm 0.2$ & -0.573 & 0.57 \\
\hline Thyroid-stimulating hormone $(\mu \mathrm{IU} / \mathrm{mL})$ & $1.6 \pm 0.7$ & $1.6 \pm 0.9$ & -0.141 & 0.888 \\
\hline Hemoglobin $(\mathrm{g} / \mathrm{dL})$ & $13.3 \pm 1.0$ & $13.6 \pm 0.9$ & -1.111 & 0.273 \\
\hline Hematocrit (\%) & $40.3 \pm 2.6$ & $41.1 \pm 2.1$ & -1.069 & 0.291 \\
\hline $\mathrm{RBC}$ count $\left(10^{6} / \mu \mathrm{L}\right)$ & $4.4 \pm 0.4$ & $4.5 \pm 0.3$ & -1.384 & 0.174 \\
\hline WBC count $\left(10^{3} / \mu \mathrm{L}\right)$ & $5.7 \pm 1.8$ & $7.1 \pm 1.8$ & -2.408 & 0.021 \\
\hline Platelet count $\left(10^{3} / \mu \mathrm{L}\right)$ & $268.1 \pm 58.8$ & $279.2 \pm 46.7$ & -0.668 & 0.508 \\
\hline
\end{tabular}

TABLE 3: Comparison of serum cytokine levels using multiplex cytokine assays.

\begin{tabular}{|c|c|c|c|c|}
\hline Serum cytokine levels $(\mathrm{pg} / \mathrm{mL})$ & Normal $(n=21$, female $)$ & Obese $(n=20$, female $)$ & $t$ & $P$ \\
\hline C-peptide & $1673.2 \pm 787.3$ & $3395.5 \pm 1319.7$ & -5.104 & $<0.001$ \\
\hline GIP & $64.4 \pm 54.3$ & $102.5 \pm 203.1$ & -0.831 & 0.411 \\
\hline Insulin & $27.7 \pm 13.7$ & $52.4 \pm 24.9$ & -3.906 & 0.001 \\
\hline Leptin & $842.0 \pm 706.2$ & $3498.7 \pm 1612.0$ & -6.777 & $<0.001$ \\
\hline MCP-1 & $1163.7 \pm 493.1$ & $1313.7 \pm 430.1$ & -1.036 & 0.307 \\
\hline $\mathrm{PP}$ & $110.8 \pm 61.8$ & $119.2 \pm 72.1$ & -0.402 & 0.69 \\
\hline IL-8 & $2098.1 \pm 2500.2$ & $1637.5 \pm 3468.3$ & 0.49 & 0.627 \\
\hline HGF & $174.1 \pm 84.0$ & $285.3 \pm 160.8$ & -2.753 & 0.01 \\
\hline Adiponectin & $10228.4 \pm 1709.2$ & $8732.5 \pm 1748.4$ & 2.77 & 0.009 \\
\hline Lipocalin-2 & $3429.9 \pm 1335.8$ & $4552.5 \pm 2086.3$ & -2.062 & 0.046 \\
\hline Resistin & $807.3 \pm 530.7$ & $1048.1 \pm 669.4$ & -1.28 & 0.208 \\
\hline Adipsin & $16287.9 \pm 1044.8$ & $17351.4 \pm 752.5$ & -3.723 & 0.001 \\
\hline Total PAI-1 & $1953.5 \pm 647.3$ & $2701.9 \pm 743.7$ & -3.441 & 0.001 \\
\hline
\end{tabular}

significantly correlated with HGF, lipocalin-2, adipsin, and total PAI-1. PP was significantly correlated with adiponectin, resistin, and total PAI-1. IL-8 was significantly correlated with lipocalin-2. HGF was significantly correlated with lipocalin2, resistin, adipsin, and total PAI-1. Lipocalin-2 was significantly correlated with resistin and adipsin. Resistin was significantly correlated with adipsin. However, MCP-1 was not significantly correlated with any other cytokines.

Table 5 presents that mean serum leptin of Eui-E-In-Tang group was found to be significantly reduced by Eui-E-In-Tang therapy for 12 weeks $(P=0.037)$. In contrast, Eui-E-In-Tang did not alter serum levels of C-peptide, GIP, insulin, MCP-1, 
TABLE 4: Spearman correlation analysis of cytokines.

\begin{tabular}{|c|c|c|c|c|c|c|c|c|c|c|c|c|c|}
\hline & C-peptide & GIP & Insulin & Leptin & MCP-1 & $\mathrm{PP}$ & IL-8 & HGF & Adiponectin & Lipocalin-2 & Resistin & Adipsin & Total PAI-1 \\
\hline C-peptide & 1 & 0.306 & $0.852^{* *}$ & $0.651^{* *}$ & -0.029 & -0.017 & $-0.328^{*}$ & $0.418^{* *}$ & $-0.407^{* *}$ & 0.274 & 0.176 & $0.318^{*}$ & $0.634^{* *}$ \\
\hline GIP & & 1 & $0.409^{* *}$ & 0.261 & -0.232 & 0.033 & -0.153 & 0.084 & $0.312^{*}$ & $0.364^{*}$ & -0.017 & 0.179 & 0.273 \\
\hline Insulin & & & 1 & $0.583^{* *}$ & -0.036 & -0.089 & -0.275 & $0.471^{* *}$ & -0.201 & 0.287 & 0.111 & $0.374^{*}$ & $0.587^{* *}$ \\
\hline Leptin & & & & 1 & 0.158 & 0.075 & -0.082 & $0.536^{* *}$ & -0.282 & $0.322^{*}$ & 0.12 & $0.414^{* *}$ & $0.615^{* *}$ \\
\hline MCP-1 & & & & & 1 & 0.115 & 0.052 & 0.083 & -0.007 & -0.15 & -0.12 & -0.078 & 0.06 \\
\hline PP & & & & & & 1 & -0.027 & -0.063 & $0.35^{*}$ & -0.04 & $0.409^{* *}$ & 0.093 & $0.383^{*}$ \\
\hline IL-8 & & & & & & & 1 & 0.276 & 0.155 & $0.422^{* *}$ & 0.289 & -0.085 & -0.272 \\
\hline HGF & & & & & & & & 1 & -0.302 & $0.635^{* *}$ & $0.358^{*}$ & $0.484^{* *}$ & $0.422^{* *}$ \\
\hline Adiponectin & & & & & & & & & 1 & -0.032 & -0.033 & -0.119 & -0.041 \\
\hline Lipocalin-2 & & & & & & & & & & 1 & $0.609^{* *}$ & $0.346^{*}$ & 0.223 \\
\hline Resistin & & & & & & & & & & & 1 & $0.407^{* *}$ & 0.296 \\
\hline Adipsin & & & & & & & & & & & & 1 & 0.284 \\
\hline Total PAI-1 & & & & & & & & & & & & & 1 \\
\hline
\end{tabular}

${ }^{*} P<0.05$.

${ }^{* *} P<0.01$.

TABLE 5: Effect of Eui-E-In-Tang on serum cytokine levels.

\begin{tabular}{|c|c|c|c|c|c|}
\hline Serum cytokine levels (pg/mL) & Study group & $N$ & Baseline & Endpoint & $P$ \\
\hline \multirow{2}{*}{ C-peptide } & Obese-Eui-E-In-Tang & 9 & $3290.7 \pm 1194.0$ & $3481.1 \pm 1466.4$ & 0.832 \\
\hline & Obese-placebo & 11 & $3156.3 \pm 1444.6$ & $3129.7 \pm 2203.9$ & 0.664 \\
\hline \multirow{2}{*}{ GIP } & Obese-Eui-E-In-Tang & 9 & $76.3 \pm 90.5$ & $124.0 \pm 265.9$ & 0.703 \\
\hline & Obese-placebo & 11 & $63.0 \pm 48.9$ & $58.6 \pm 51.9$ & 0.433 \\
\hline \multirow{2}{*}{ Insulin } & Obese-Eui-E-In-Tang & 9 & $57.9 \pm 24.2$ & $47.8 \pm 25.6$ & 0.262 \\
\hline & Obese-placebo & 11 & $46.3 \pm 17.7$ & $91.9 \pm 137.8$ & 0.309 \\
\hline \multirow{2}{*}{ Leptin } & Obese-Eui-E-In-Tang & 9 & $3749.5 \pm 1556.7$ & $3293.5 \pm 1701.6$ & 0.037 \\
\hline & Obese-placebo & 11 & $2281.4 \pm 1148.6$ & $2856.6 \pm 1157.4$ & 0.489 \\
\hline \multirow{2}{*}{ MCP-1 } & Obese-Eui-E-In-Tang & 9 & $1231.0 \pm 381.9$ & $1381.4 \pm 473.0$ & 0.471 \\
\hline & Obese-placebo & 11 & $1409.2 \pm 615.2$ & $1462.4 \pm 760.0$ & 0.767 \\
\hline \multirow{2}{*}{$\mathrm{PP}$} & Obese-Eui-E-In-Tang & 9 & $131.0 \pm 100.4$ & $109.5 \pm 39.8$ & 0.365 \\
\hline & Obese-placebo & 11 & $193.7 \pm 175.0$ & $161.4 \pm 196.7$ & 0.401 \\
\hline \multirow{2}{*}{ IL-8 } & Obese-Eui-E-In-Tang & 9 & $954.3 \pm 1610.5$ & $2196.5 \pm 4474.1$ & 0.231 \\
\hline & Obese-placebo & 11 & $2473.8 \pm 3287.8$ & $3437.3 \pm 3850.0$ & 0.494 \\
\hline \multirow{2}{*}{ HGF } & Obese-Eui-E-In-Tang & 9 & $299.7 \pm 162.5$ & $273.5 \pm 166.4$ & 0.373 \\
\hline & Obese-placebo & 11 & $240.3 \pm 106.9$ & $268.5 \pm 127.4$ & 0.938 \\
\hline \multirow{2}{*}{ Adiponectin } & Obese-Eui-E-In-Tang & 9 & $8954.3 \pm 1676.9$ & $8551.0 \pm 1864.9$ & 0.697 \\
\hline & Obese-placebo & 11 & $9219.6 \pm 1097.8$ & $8470.6 \pm 1669.0$ & 0.916 \\
\hline \multirow{2}{*}{ Lipocalin-2 } & Obese-Eui-E-In-Tang & 9 & $4239.4 \pm 1258.6$ & $4808.5 \pm 2615.8$ & 0.323 \\
\hline & Obese-placebo & 11 & $3465.1 \pm 1897.7$ & $4050.4 \pm 1833.9$ & 0.440 \\
\hline \multirow{2}{*}{ Resistin } & Obese-Eui-E-In-Tang & 9 & $1163.2 \pm 763.1$ & $953.9 \pm 603.3$ & 0.473 \\
\hline & Obese-placebo & 11 & $940.7 \pm 493.1$ & $821.5 \pm 415.1$ & 0.556 \\
\hline \multirow{2}{*}{ Adipsin } & Obese-Eui-E-In-Tang & 9 & $17645.9 \pm 591.6$ & $17110.3 \pm 808.6$ & 0.751 \\
\hline & Obese-placebo & 11 & $17553.3 \pm 624.6$ & $17067.4 \pm 894.1$ & 0.907 \\
\hline \multirow{2}{*}{ Total PAI-1 } & Obese-Eui-E-In-Tang & 9 & $2867.1 \pm 866.5$ & $2566.7 \pm 636.9$ & 0.438 \\
\hline & Obese-placebo & 11 & $2556.1 \pm 789.0$ & $2858.0 \pm 886.5$ & 0.387 \\
\hline
\end{tabular}


PP, IL-8, HGF, adiponectin, lipocalin-2, resistin, adipsin, and total PAI-1 $(P>0.05)$. Table 5 also presents that the serum cytokine levels of obese group with the placebo capsules for 12 weeks do not show any significant variation $(P>0.05)$.

\section{Discussion}

Overweight and obesity are defined as abnormal or excessive fat accumulation that may impair health by the WHO definition [1]. Obesity is a global health problem and is associated with chronic inflammation, which is linked to many diseases including diabetes, cardiovascular diseases, metabolic syndrome, and gout. According to the WHO statistics, about $13 \%$ of the world's adult population (11\% of men and 15\% of women) was obese in 2014 [1].

Ghayour-Mobarhan et al. have reported that obesity is associated with a strong inflammatory response and is often accompanied by increased levels of proinflammatory cytokines and impaired antioxidant status [12]. Spite et al. have reported that although inflammation is thought to be essential for reacting to pathologic infection, ungoverned inflammation is an underlying component of various diseases, such as sepsis, cardiovascular disease, diabetes, and other chronic inflammatory diseases [13]. Maskrey et al. have reported that chronic inflammation is a characteristic feature in virtually all inflammatory diseases, including cardiovascular disease such as atherosclerosis, and it is becoming increasingly clear that disturbance of the processes usually involved in resolution of inflammation is an underlying feature of chronic inflammatory conditions [14]. Skeldon et al. have reported that inflammation also is regarded as an important contributor to the development of metabolic disease and recent work has strongly implicated the inflammasome as having a pivotal role in the regulation of metabolism, obesity, insulin resistance, and cardiovascular disease [15].

It is well known that cytokine is an important mediator in acute or chronic inflammation. Burska et al. have reported that cytokines are small proteins which play important roles in cell signaling and they are secreted by a variety of cellular sources acting either on the cell producing them (autocrine) or on the surrounding cells (paracrine) [16]. Nakagawa et al. have reported that IL-6 is a multifunctional cytokine that is produced by many different cell types, and plays an important role in the regulation of inflammation, immune responses, the acute-phase response, and hematopoiesis [17]. Niebler et al. have reported that IL-1 $\beta$ is also included in central key players in the immune surveillance interactome, which not only mediates inflammation but also links innate and adaptive immunity [18]. Tracey et al. have reported that TNF$\alpha$ is a pleiotropic cytokine known to play a major role in host defense mechanisms, initiating a beneficial local inflammation which in excess, however, may cause tissue damage concerned with the pathogenesis of numerous autoimmune and/or inflammatory systemic diseases [19].

Cao has reported that obesity induces production of inflammatory cytokines (often referred to together with adipokines as adipocytokines) and infiltration of immune cells into adipose tissue, which creates a state of chronic lowgrade inflammation [20]. Ganjali et al. have reported that the most important source of proinflammatory cytokines in obesity is macrophages that infiltrate adipose tissue as a response to the adipocyte growth, decreased blood supply, hypoxia, and tissue necrosis [21]. Blüher and Mantzoros have reported that the adipose tissue influences the regulation of several important physiological functions including but not limited to appetite, satiety, energy expenditure, activity, insulin sensitivity and secretion, glucose and lipid metabolism, fat distribution, endothelial function, hemostasis, blood pressure, neuroendocrine regulation, and function of the immune system through adipokines [9]. On the other hand, Park et al. have reported that Euiiin-tang (yiyiren-tang; Eui-E-In-Tang) granules exert anti-inflammation and antiobesity effect on high fat diet-induced obese C57 BL/6J mice [22].

These days, the number of reports for adipokines with obesity is increasing. Leptin is well known to be a forerunner of the adipokine superfamily. Park and Ahima have reported that leptin is secreted by adipose tissue and regulates energy homeostasis, neuroendocrine function, metabolism, immune function, and other systems through its effects on the central nervous system and peripheral tissues [8].

In order to examine the inflammatory mediators of obesity and determine a possible distinctive inflammatory blood marker, we measured the serum levels of 21 cytokines using multiplex cytokine assay. Unlike infectious inflammation diseases increase the serum levels of proinflammatory cytokines such as IL-1 $\beta$, IL-6, TNF- $\alpha$, and MCP-1, our data represent that the serum C-peptide, insulin, leptin, HGF, lipocalin, adipsin, and total PAI-1 levels are higher in the obese group than the normal group; the serum adiponectin level is lower in the obese group than the normal group. After adjusting for age, height, SGPT, alkaline phosphatase, GGTP, total cholesterol, HDL cholesterol, triglyceride, uric acid, glucose, CRP, and WBC, the serum C-peptide, insulin, leptin, lipocalin-2, and adipsin levels in the obese group were found to be significantly higher than in the normal group. C-peptide was significantly correlated with insulin, leptin, IL-8, HGF, adiponectin, adipsin, and total PAI-1. Insulin was significantly correlated with C-peptide, GIP, leptin, HGF, adipsin, and total PAI-1. Leptin was significantly correlated with C-peptide, insulin, HGF, lipocalin-2, adipsin, and total PAI-1. Lipocalin-2 was significantly correlated with GIP, leptin, IL-8, HGF, resistin, and adipsin. Adipsin was significantly correlated with C-peptide, insulin, leptin, HGF, lipocalin-2, and resistin. How correlations of cytokines are varied and involved in obesity deserves further investigation in cells, animal models, and human populations.

And the investigation of serum cytokine levels at the end point of this study demonstrated that mean serum leptin of Eui-E-In-Tang group was found to be significantly reduced by Eui-E-In-Tang therapy for 12 weeks. In contrast, Eui-EIn-Tang therapy did not show any significant effect on the concentrations of C-peptide, insulin, lipocaline-2, adipsin, HGF, total PAI-1, GIP, MCP-1, PP, IL-8, adiponectin, and resistin. The findings on the modulatory effect of Eui-E-InTang on serum level of leptin deserve to be studied further to make a safe and effective treatment for obesity.

We acknowledge limitations in our study. The most important limitation relates to the small sample size. Another 
limitation of the present study was the lack of gender diversity in the study group. It is recommended that further studies be designed for a large sample size including male individuals.

\section{Competing Interests}

The authors declare that there is no conflict of interests regarding the publication of this paper.

\section{Acknowledgments}

The authors would like to acknowledge Professor Seong-Gyu Ko (College of Korean Medicine, Kyung Hee University), Dr. Young-Jin Kim, Hyun Joo Kim, and Dr. Min-Youn Ahn (College of Korean Medicine, Gachon University) for their valuable help in this study. The authors would like to thank all the individuals who participated in the study. This research was supported by the Basic Science Research Program through the National Research Foundation of Korea, funded by the Ministry of Education, Science and Technology (20100022919), and by a grant of the Oriental Medicine R\&D Program, funded by the Ministry of Health, Welfare \& Family Affairs, Republic of Korea (B110068-1101-0000200).

\section{References}

[1] World Health Organization, "Obesity and overweight," Fact Sheet N311, World Health Organization, Geneva, Switzerland, 2014, http://www.who.int/mediacentre/factsheets/fs311/en/.

[2] Y. Peng, S. Yu, H. Li, H. Xiang, J. Peng, and S. Jiang, "MicroRNAs: emerging roles in adipogenesis and obesity," Cellular Signalling, vol. 26, no. 9, pp. 1888-1896, 2014.

[3] V. Contreras-Shannon, D. L. Heart, R. M. Paredes et al., "Clozapine-induced mitochondria alterations and inflammation in brain and insulin-responsive cells," PLOS ONE, vol. 8, no. 3, Article ID e59012, 2013.

[4] C. Robertson, D. Archibald, A. Avenell et al., "Systematic reviews of and integrated report on the quantitative, qualitative and economic evidence base for the management of obesity in men," Health Technology Assessment, vol. 18, no. 35, 2014.

[5] R. Adya, B. K. Tan, and H. S. Randeva, "Differential effects of leptin and adiponectin in endothelial angiogenesis," Journal of Diabetes Research, vol. 2015, Article ID 648239, 12 pages, 2015.

[6] G. dos Santos, M. A. Kutuzov, and K. M. Ridge, "The inflammasome in lung diseases," American Journal of Physiology —Lung Cellular and Molecular Physiology, vol. 303, no. 8, pp. L627L633, 2012.

[7] M. Alvehus, J. Burén, M. Sjöström, J. Goedecke, and T. Olsson, "The human visceral fat depot has a unique inflammatory profile," Obesity, vol. 18, no. 5, pp. 879-883, 2010.

[8] H.-K. Park and R. S. Ahima, "Physiology of leptin: energy homeostasis, neuroendocrine function and metabolism," Metabolism: Clinical and Experimental, vol. 64, no. 1, pp. 24-34, 2015.

[9] M. Blüher and C. S. Mantzoros, "From leptin to other adipokines in health and disease: facts and expectations at the beginning of the 21st century," Metabolism: Clinical and Experimental, vol. 64, no. 1, pp. 131-145, 2015.
[10] C.-L. Yen, W.-C. Chao, C.-H. Wu et al., "Phosphorylation of glycogen synthase kinase- $3 \beta$ in metabolically abnormal obesity affects immune stimulation-induced cytokine production," International Journal of Obesity, vol. 39, no. 2, pp. 270-278, 2015.

[11] W. Schulte, J. Bernhagen, and R. Bucala, "Cytokines in sepsis: potent immunoregulators and potential therapeutic targets-an updated view," Mediators of Inflammation, vol. 2013, Article ID 165974, 16 pages, 2013.

[12] M. Ghayour-Mobarhan, A. Sahebkar, S. M. R. Parizadeh et al., "Antibody titres to heat shock protein 27 are elevated in patients with acute coronary syndrome," International Journal of Experimental Pathology, vol. 89, no. 3, pp. 209-215, 2008.

[13] M. Spite, L. V. Norling, L. Summers et al., "Resolvin D2 is a potent regulator of leukocytes and controls microbial sepsis," Nature, vol. 461, no. 7268, pp. 1287-1291, 2009.

[14] B. H. Maskrey, I. L. Megson, P. D. Whitfield, and A. G. Rossi, "Mechanisms of resolution of inflammation: a focus on cardiovascular disease," Arteriosclerosis, Thrombosis, and Vascular Biology, vol. 31, no. 5, pp. 1001-1006, 2011.

[15] A. M. Skeldon, M. Faraj, and M. Saleh, "Caspases and inflammasomes in metabolic inflammation," Immunology and Cell Biology, vol. 92, no. 4, pp. 304-313, 2014.

[16] A. Burska, M. Boissinot, and F. Ponchel, "Cytokines as biomarkers in rheumatoid arthritis," Mediators of Inflammation, vol. 2014, Article ID 545493, 24 pages, 2014.

[17] H. Nakagawa, T. Tamura, Y. Mitsuda et al., "Inverse correlation between serum interleukin-6 and iron levels among Japanese adults: a cross-sectional study," BioMed Central Hematology, vol. 14, no. 1, p. 6, 2014.

[18] M. Niebler, X. Qian, D. Höfler et al., "Post-translational control of IL-1 $\beta$ via the human papillomavirus type 16 E6 oncoprotein: a novel mechanism of innate immune escape mediated by the E3-ubiquitin ligase E6-AP and p53," PLoS Pathogens, vol. 9, no. 8, Article ID e1003536, 2013.

[19] D. Tracey, L. Klareskog, E. H. Sasso, J. G. Salfeld, and P. P. Tak, "Tumor necrosis factor antagonist mechanisms of action: a comprehensive review," Pharmacology \& Therapeutics, vol. 117, no. 2, pp. 244-279, 2008.

[20] H. Cao, "Adipocytokines in obesity and metabolic disease," The Journal of Endocrinology, vol. 220, no. 2, pp. T47-T59, 2014.

[21] S. Ganjali, A. Sahebkar, E. Mahdipour et al., "Investigation of the effects of curcumin on serum cytokines in obese individuals: a randomized controlled trial," The Scientific World Journal, vol. 2014, Article ID 898361, 6 pages, 2014.

[22] K. M. Park, H. H. Lim, and Y. K. Song, "Anti-inflammation and anti-obesity effects of Euiiin-tang granules on high fat diet-induced obese C57BL/6J mice," Journal of Korean Medical Rehabilitation, vol. 22, no. 2, pp. 47-66, 2012. 


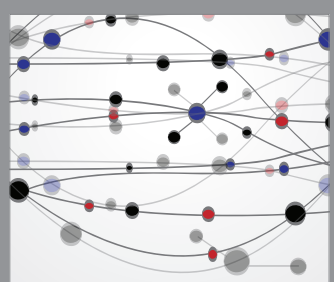

The Scientific World Journal
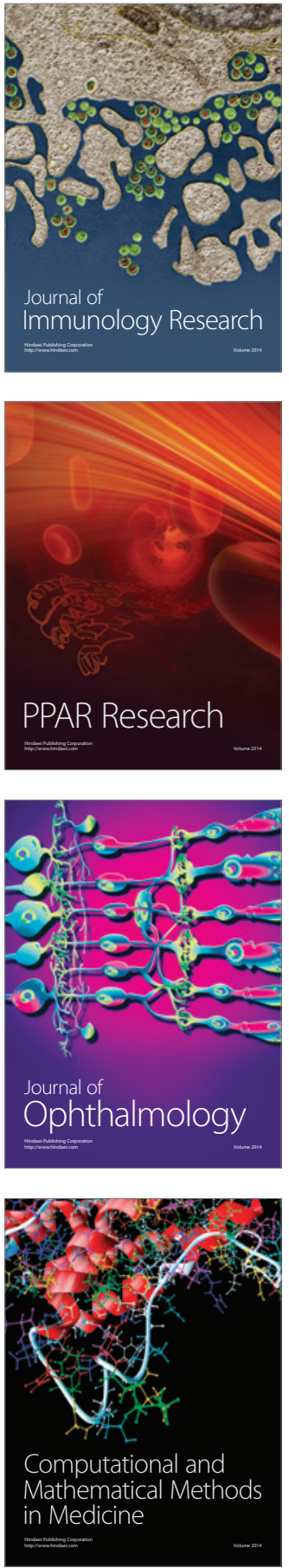

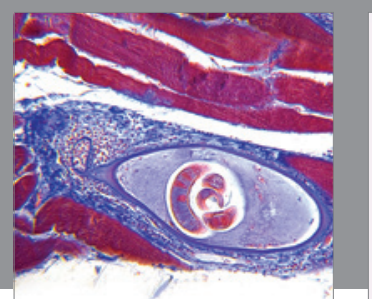

Gastroenterology Research and Practice

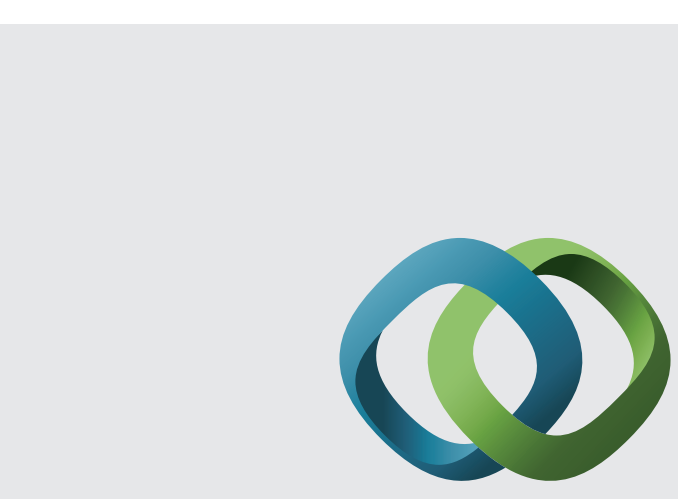

\section{Hindawi}

Submit your manuscripts at

http://www.hindawi.com
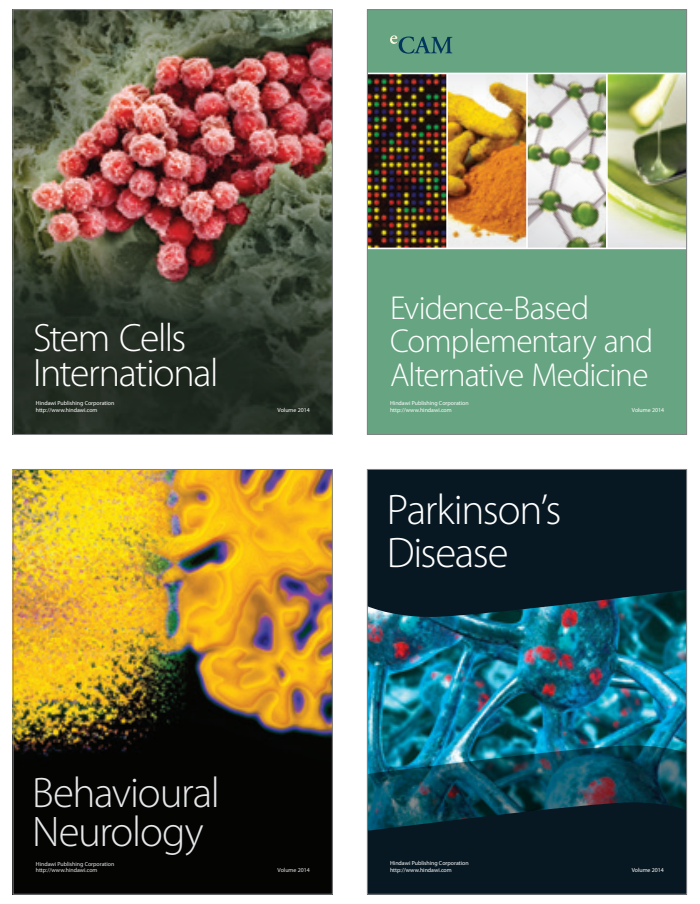
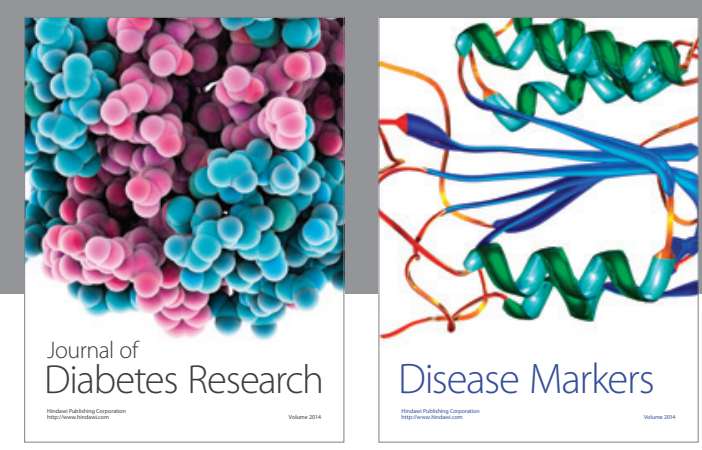

Disease Markers
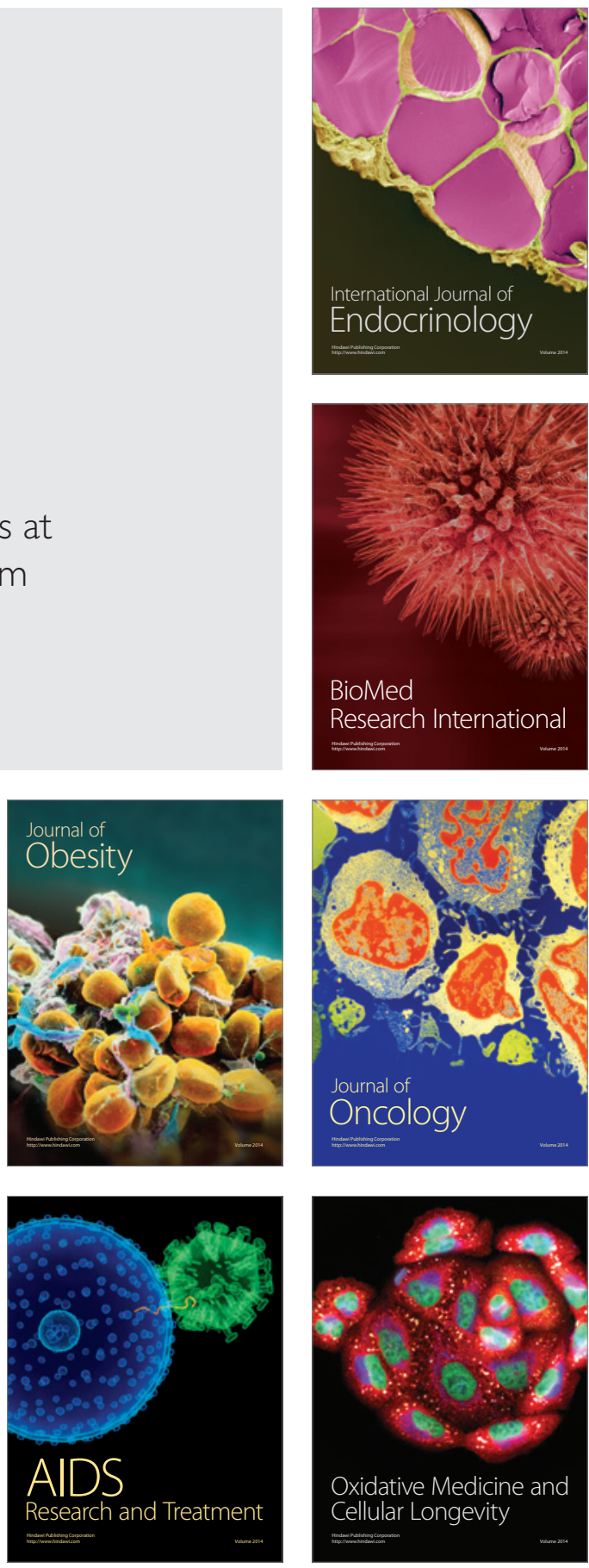